Originalveröffentlichung in: Grüne, Niels ; Oberhauser, Claus (Hrsgg.): Jenseits des

Illustrativen : visuelle Medien und Strategien politischer Kommunikation, Göttingen 2015, S. 39-60 (Schriften zur politischen Kommunikation ; 20)

Martina Sauer

\title{
Visualität und Geschichte. Bilder als historische Akteure im Anschluss an Verkörperungstheorien
}

\section{Einleitung}

Können Bilder ebenso wie sprachliche Äußerungen handlungsrelevant sein? Lassen sie sich als wichtige Faktoren unserer gesellschaftlichen Wirklichkeit ausmachen und von daher als historische Akteure verstehen?

Ein deutlicher Hinweis darauf, dass Bilder tatsächlich diese Funktion übernehmen können, ist die Tatsache, dass wir Bilder nicht nur selbst produzieren und verbreiten, sondern auch je nach Kontext und Absicht verändern und damit neue Akzente setzen. Mit Bildern, so lässt sich daraus schließen, können wir tatsächlich kommunizieren und darüber hinaus Einfluss auf Wertsetzungen und damit auf Entscheidungen und Handlungen nehmen. In den Forschungen zur visual history, wie sie seit Anfang der neunziger Jahre mit dem pictorial turn durch William J. T. Mitchell initiiert wurden, spiegelt sich die Auseinandersetzung mit diesem Phänomen wider. ${ }^{1}$ Doch vor dem Hintergrund der Fragestellung Mitchells »what do pictures want? « spielt die Frage, ob und wie wir konkret über die Bilder selbst kommunizieren können, keine Rolle. ${ }^{2}$ Ursprünglich war es parallel zu Mitchell der Bildwissenschaftler Gottfried Boehm, der sich dieser Frage hätte annehmen können, indem er mit dem iconic turn auf die Eigenlogik der Bilder verwies. Konkrete lebensweltliche Bezüge arbeitete er jedoch nicht heraus. ${ }^{3}$ Hoffnungen darauf, die Relevanz von Bildern als historischen Akteuren aufzuzeigen, vermittelte 2006 der Vortrag des Kunsthistorikers Horst Brede-

1 Vgl. Gerhard Paul, BilderMACHT. Studien zur Visual History des 20. und 21. Jahrhunderts, Göttingen 2013.

2 William J. T. Mitchell, What Do Pictures Want? The Lives and Loves of Images, Chicago 2005.

3 Stattdessen setzte Boehm mit der Betonung einer "Geschichte des Sehens" eigene Akzente. Demnach vermittle das Bild in jedem Jahrhundert anders etwas vom "Ur-Bild, der Grenze oder Spur«. Vgl. hierzu grundlegend Gottfried Boehm, $\mathrm{Zu}$ einer Hermeneutik des Bildes, in: ders. / Hans-Georg Gadamer (Hg.), Seminar: Die Hermeneutik und die Wissenschaften, 2. Aufl., Frankfurt a. M. 1985, S. 444-471, hier S. 454; ders., Augenmaß. Zur Genese der ikonischen Evidenz, in: ders. / Birgit Mersmann / Christian Spies (Hg.), Movens Bild. Zwischen Evidenz und Affekt, München 2008, S. 15-38, hier S. 21 ff. 
kamp auf dem 46. Deutschen Historikertag in Konstanz. ${ }^{4}$ Doch eine operationalisierbare Lösung, wie sich Bilder so analysieren lassen, dass deren Handlungskraft hervortritt, zeichnete sich mit Bredekamps mit Spannung erwarteter "Theorie des Bildakts" (2010) nicht ab, wie der Historiker Jens Jäger in seiner Rezension verdeutlichte. ${ }^{5}$

Bilder als Mittel der Kommunikation zu verstehen, hat jedoch - diesem ersten Befund entgegen - innerhalb der Kunstgeschichte eine lange Tradition. Sie geht auf deren Anfänge im 19. Jahrhundert zurück, als neben der Analyse des historisch gesicherten Gehalts die Aufarbeitung der stilistischen Eigenarten bzw. der Logik der Bilder im Vordergrund stand. Die formale Ästhetik machte deutlich, dass eine Trennung von Form und Inhalt nicht möglich ist. Der Münchner Kunsthistoriker Hermann Bauer brachte diesen Zusammenhang in einer Anthologie zu den Methoden des Fachs 1985 auf den Punkt:

»Sollte die Minimal-Definition von der Form als der Gestalt der Mitteilung richtig sein, ergibt sich, daß eine reine Formanalyse unmöglich ist, weil es unmöglich ist, die Mitteilung selbst nicht $\mathrm{zu}$ berühren. Nur von einer Mitteilung zu sprechen, geht ebensowenig an, denn die Mitteilung existiert nur in einer bestimmten Form. ${ }^{6}$

Diesem Befund entspricht die Aufarbeitung der Geschichte und Grundlagen der formanalytischen und formgeschichtlichen Methoden durch den Philosophen Lambert Wiesing. ${ }^{7}$ Demnach war es erstmals Heinrich Wölfflin, der 1915 auf eine Analogie zwischen den Gestaltungsweisen (Form / Stil) und den Anschauungsweisen (Inhalt / Mitteilung) von Bildern hinwies. ${ }^{8}$ Implizit wird damit unterstellt, dass es einen Weg der Übertragung von Anschauungsweisen (des Gestalters) in Gestaltungsweisen gibt, dessen Sinngehalt von einem Betrachter verstanden und damit auch handlungsrelevant werden kann. Wie ist das möglich?

4 Horst Bredekamp, Schlussvortrag: Bild - Akt - Geschichte, in: Clemens Wischermann u. a. (Hg.), GeschichtsBilder. 46. Deutscher Historikertag vom 19. bis 22. September in Konstanz. Berichtsband, Konstanz 2007, S. 289-309.

5 Horst Bredekamp, Theorie des Bildakts. Frankfurter Adornovorlesungen, Frankfurt a. M. 2010. Vgl. hierzu die Rezension von Jens Jäger in: H-Soz-u-Kult, 14.7.2011; http://hsozkult. geschichte.hu-berlin.de/rezensionen/2011-3-037 [5.9.2014]. Entgegen diesem ersten Befund erweist sich jedoch Bredekamps Initiative zur Gründung des breit aufgestellten, interdisziplinären Labors "Bild - Wissen - Gestaltung" an der Humboldt-Universität Berlin seit Mitte 2012 als sehr vielversprechend. Aus unterschiedlichen Perspektiven werden darin Gestaltungsprozesse in den Wissenschaften untersucht: https://www.interdisciplinary-laboratory. hu-berlin.de/de.

6 Hermann Bauer, Form, Struktur, Stil: Die formanalytischen und formgeschichtlichen Methoden, in: Hans Belting u. a. (Hg.), Kunstgeschichte. Eine Einführung, 3., durchges. u. erw. Aufl., Berlin 1988, S. 151-168, hier S. 158.

7 Lambert Wiesing, Die Sichtbarkeit des Bildes. Geschichte und Perspektiven der formalen Ästhetik (1997), Frankfurt a. M. 2008.

8 Ebd., S. $16 \mathrm{ff}$. 
Es sind die kulturwissenschaftlichen Symbol- und Verkörperungstheorien in der Nachfolge Ernst Cassirers und des amerikanischen Pragmatismus von Susanne K. Langer und John M. Krois sowie ergänzend entwicklungspsychologische und neurowissenschaftliche Forschungen, die Antworthorizonte erkennbar werden lassen und zu einem neuen Ansatz inspirieren, den es im Folgenden vorzustellen gilt. So soll die Auswertung ihrer Forschungen zur Frage des $\mathrm{Zu}$ sammenhangs von Form und Inhalt deutlich machen, dass er nicht nur von sprachlichen Prozessen geleistet wird, ${ }^{9}$ sondern zunächst auf körpereigenen Prozessen beruht. ${ }^{10}$ Diese Annahme eröffnet ganz neue Perspektiven, denn gerade das sprachlich-sachlich feststellende Erfassen der Bildmotive vermag kaum Bewertungsprozesse anzustoßen, die als Grundlage für Entscheidungen und Handlungen angesehen werden können. Das gilt auch dann, wenn die Motive selbst weiterführende Bedeutungen für uns haben. Dafür ist vielmehr die Relevanz für Zukünftiges, die der Übertragungsvorgang des Verstehens vermitteln muss, grundlegend. ${ }^{11}$ Das angenommene Bewertungsverfahren bzw. der Meinungsbildungsprozess, zu dem Bilder anregen, entzündet sich entsprechend nicht an den Darstellungsinhalten (Was), sondern an den Darstellungsweisen (Wie) und damit an der jeweiligen formalen Struktur der Bilder. So sind es die Strukturen selbst bzw. die je individuell gesetzten Striche, Farben, Flächen und die mit ihnen hergestellte Komposition, die bewertet werden. Bewerten meint hier, dass die bildnerischen Elemente nicht als neutrale Faktoren aufgenommen, sondern ihre jeweilige Erscheinungsweise für den Betrachter affektiv-emotional relevant wird. In Abgrenzung zu der noch von Cassirer, Langer und Krois vertretenen traditionellen Auffassung, dass diese Erfahrung das spezifische ästhetische Erlebnis mit Bildern bzw. Kunst ausmache (ästhetische Theorie), gilt es im Folgenden die kommunikative Funktion von Bildern aufzuzeigen. Demnach vermittelt sich über die lebendige Wirkung, die Bewertung (die Ansicht / Meinung) des Gestalters / Künstlers ein Motiv, auf das der Betrachter antworten kann (bildsemiotischer Ansatz).

9 Eine Annahme, die zuletzt Lambert Wiesing mit seinem jüngsten Buch vertritt: ders., Sehen lassen. Die Praxis des Zeigens, Berlin 2013, bes. S. $211 \mathrm{f}$.

10 Diese Annahme baut auf ersten Untersuchungen von mir zum Thema auf. Vgl. zuletzt: Martina Sauer, Ästhetik und Pragmatismus. Zur funktionalen Relevanz einer nicht-diskursiven Formauffassung bei Cassirer, Langer und Krois, in: IMAGE. Zeitschrift für interdisziplinäre Bildwissenschaft 20 (2014); http://www.gib.uni-tuebingen.de/image?function= fnArticle\& showArticle $=303$ [5.9.2014].

11 In bemerkenswerter Klarheit wird dieser Zusammenhang von dem Lehrer Susanne K. Langers, dem Philosophen des Pragmatismus Alfred North Whitehead in dessen Vorlesung 1927 zum Thema "Symbolic Expression: Its Function for the Individual and for Society" ausgearbeitet. Sie wurde von Rolf Lachmann übersetzt und unter dem Titel »Kulturelle Symbolisierung « herausgegeben. Vgl. ders. (Hg.), Alfred North Whitehead. Kulturelle Symbolisierung, Frankfurt a. M. 2007, S. 101-108. 


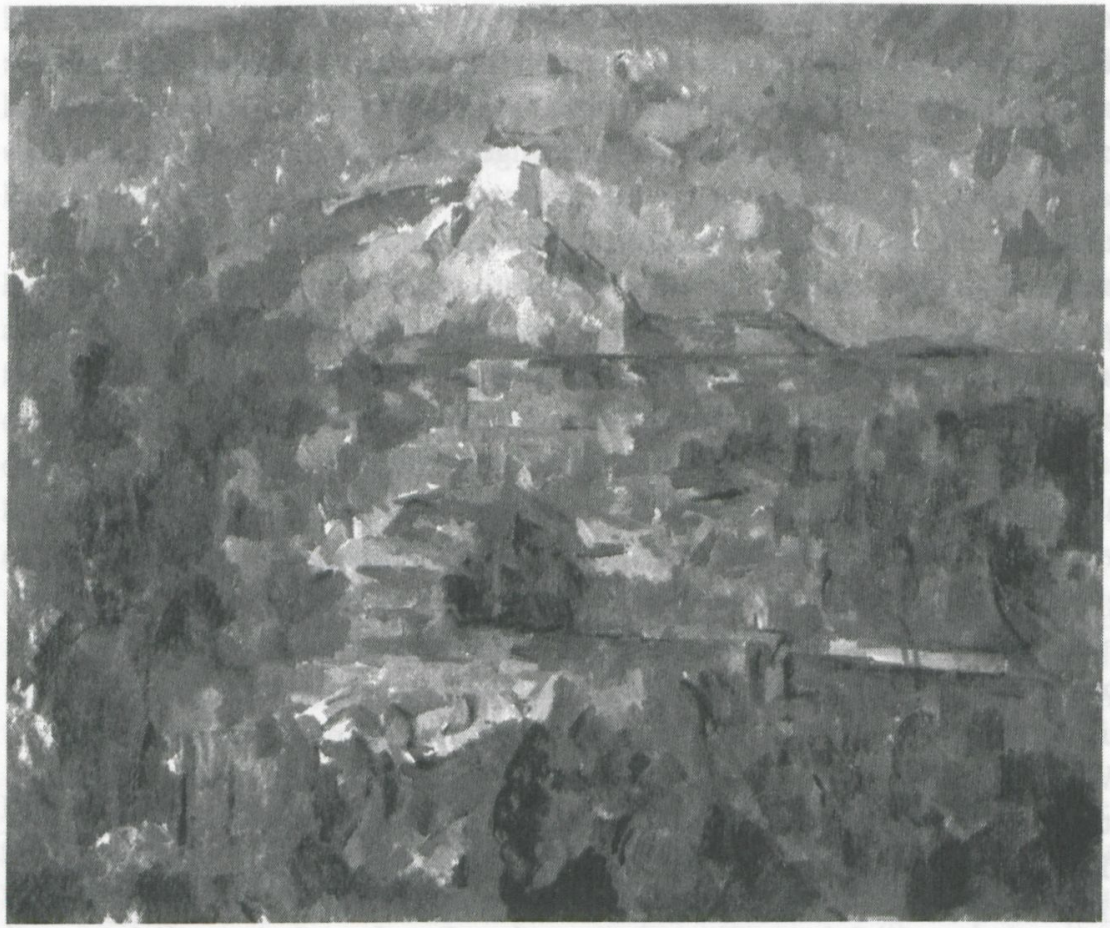

Abb. 1: Paul Cézanne, "Montagne Ste. Victoire«, 1904/06, 60 x 72 cm, Öl auf Leinwand, Kunstmuseum Basel. Aus: Martina Sauer, Cézanne, van Gogh, Monet. Genese der Abstraktion, Bühl 2000, Anhang (Abb. 1).

Zur Verdeutlichung des Unterschieds soll hier als Beispiel ein Spätwerk Paul Cézannes, eine Fassung der »Montagne Ste. Victoire« von 1906 herangezogen werden (Abb. 1). Schnell wird deutlich: Die Details des Motivs sind wegen der vereinzelten Strichführung (taches) kaum differenzierbar, und dennoch entsteht der Eindruck von stiller Größe. Wie ist das möglich? Es handelt sich ja gerade nicht um einen Berg am See mit einem Boot. Das heißt, am Motiv kann sich diese Wertung kaum festmachen. Verständlich und nachvollziehbar wird sie jedoch, wenn auf das Erregungspotential der gleichförmig über das Bild verteilten taches geachtet wird. Stimmungsmäßig vermittelt sich über sie unendliche Ruhe. Bezogen auf den Berg, der durch den angedeuteten Umriss deutlicher hervortritt und mit seiner Stellung über der Horizontlinie einen Wechsel von der Aufsicht in die Ebene davor zu einer Untersicht veranlasst, gewinnt die Erfahrung an Bedeutung: Über das eigenen Erleben vermag dem Motiv eine gewisse Würde und Erhabenheit zugeschrieben werden. ${ }^{12}$ Hieran schließt die entscheidende Frage

12 Vgl. hierzu Martina Sauer, Cézanne, van Gogh, Monet. Genese der Abstraktion, Bühl 2000. 
an: Zeigt der Künstler nur eine lebendige Ansicht (ästhetische Theorie) oder vermittelt er seine Ansicht (Meinung) über ein Motiv (Bildsemiotik)? Hierin spiegelt sich zugleich die Doppeldeutigkeit des Begriffs "Ansicht", die theoretisch weitreichende Konsequenzen hat. In dem einen Fall zeigt sich das Wesen oder die Dichte eines Motivs (oder Themas), im anderen wird dessen Auslegung durch den Künstler deutlich. Während das ästhetische Urteil mehr oder weniger unabhängig vom Künstler erfolgt und indirekt einen Rückschluss auf dessen Können erlaubt, wird über den Einbezug des Künstlers eine Analyse der wertebildenden Prozesse (Meinungsbildung) angeregt - konkret darüber, welche Ansicht der Künstler mit dem Bild verbreitet.

Für die nachfolgende Betrachtung der Verkörperungstheorien von Cassirer, Langer und Krois ist jedoch zunächst nicht die Differenz der Auslegung dessen, was wahrgenommen wird, von Bedeutung, sondern zunächst nur die Frage nach dem Wie. Denn darin liegen die Gemeinsamkeiten sowohl mit der formalen Ästhetik als auch mit der Entwicklungspsychologie und Neurowissenschaft. Grundlegend dafür ist, dass die Gestaltung (von etwas) auf abstrakt-formalen Prinzipien aufbaut, affektiv-emotional ausgelegt und daher für die Auslegung bedeutsam wird. Entsprechend dieser Annahme besteht zwischen der Gestaltung und der Wahrnehmung eine Analogie. So kann die Auswertung der kulturanthropologischen Ansätze entscheidend dazu beitragen, die hier vertretene, bildsemiotisch orientiere These zu untermauern, die Bilder selbst als historische Akteure zu verstehen.

\section{Ausgangspunkt: Zur Handlungsrelevanz von Bildern auf der Basis körpereigener Prozesse}

Die leiblichen, affektiv-emotionalen Prozesse bei der Frage nach Bildern als historischen Akteuren in den Fokus zu rücken, erscheint auf den ersten Blick ungewöhnlich. Doch genau besehen ist diese Annahme sehr alt. Sie geht auf eine annähernd 2.500 Jahre alte Tradition zurück. Denn bereits Platon ging davon aus, dass die Künste unmittelbar auf den Rezipienten einwirken, indem sie dessen Empfindungen beeinflussen. Als rhetorische Mittel erschienen sie ihm daher äußerst suspekt. Sehr viel später war es Immanuel Kant, der ebenfalls ausdrücklich davor warnte, die Künste als "Maschinen der Überredung" einzusetzen. Möglich sei dies, wie Kant betonte, weil sie die Affekte des Menschen ansprechen und derart die reflexiven Möglichkeiten des Verstandes behindern können. Entsprechend bestanden beide Philosophen darauf, die Künste nicht

Digital auf: ART-Dok. Publikationsplattform Kunstgeschichte, 7.4.2014; http://archiv.ub. uni-heidelberg.de/artdok/2573 [5.9.2014]. 
zur Befriedigung von Bedürfnissen und Zwecken des Menschen zu missbrauchen, sondern nur diejenigen Künste gelten zu lassen, die als ein »Symbol des Sittlich-Guten" (Kant) oder als "wahre rhetorische Rede" (Platon) beurteilt werden können. ${ }^{13}$ Erst zu Beginn des 20. Jahrhunderts setzte in der Forschung ein verstärktes Interesse ein, diesen Zusammenhang näher zu ergründen.

\subsection{Entwicklungspsychologie und Neurowissenschaft}

Erste Überlegungen, weniger zur Wirkmacht von Bildern als dazu, wie überhaupt die Wahrnehmung des Menschen im Gegensatz zum Tier zu verstehen sei, wurden im Hamburger Umkreis von Ernst Cassirer in den 1920/30er Jahren entwickelt. Dazu zählte neben Aby M. Warburg, dessen Ansatz (»Pathosformeln«) hier nicht eigens aufgegriffen werden soll, auch der Entwicklungspsychologe Heinz Werner, der wie Cassirer in die USA emigrierte. In seinem lange als Standardwerk geltenden Buch »Einführung in die Entwicklungspsychologie» (1926) betonte er, dass der Mensch ursprünglich zur Welt kein distanziertes Verhältnis habe, sondern sie vielmehr als einen "vitalen Aktionszusammenhang" auffasse. Ursprünglich befinde sich der Mensch in einem Zustand einer "vitalen und affektmotorischen Totalsituation", in der Dinge pragmatisch und funktional als "Aktions- und Signaldinge", als Objektbestände eines Geschehensablaufs verstanden werden. Die Wahrnehmung einer Gestalt werde über Bewegungswahrnehmung aktiviert. Sie lasse sich bereits bei Tieren so beobachten. ${ }^{14}$ Zusammenfassend schrieb Werner: "Diese physiognomische oder ausdrucksmäßige Betrachtung der Dinge ist bedingt durch die wesentliche Mitbeteiligung des affektiven dynamischen Gesamtverhaltens an der Gegenstandsgestaltung. ${ }^{15}$ Die Welt werde daher weniger sachlich als ausdrucksmäßig, gesichthaft und lebendig erfasst. Das mache die ursprüngliche Schau von Welt aus, wie sie etwa auch Künstler haben. ${ }^{16}$

Eine entscheidende Vertiefung erfuhr dieser Ansatz in Bezug auf die Bildbzw. Kunstwahrnehmung durch den amerikanischen Entwicklungspsychologen Daniel N. Stern. ${ }^{17}$ Seine Experimente mit Säuglingen eröffneten ihm, dass bereits

13 Immanuel Kant, Kritik der Urteilskraft (1790), Stuttgart 1991, \$ 53, S. 266-273, hier S. 268. Vgl. ergänzend zu Platon die Ausarbeitung dazu von Ernesto Grassi, Macht des Bildes. Ohnmacht der rationalen Sprache. Zur Rettung des Rhetorischen, Köln 1970, S. 147-168, hier S. 166.

14 Heinz Werner, Einführung in die Entwicklungspsychologie (1926), 4., durchges. u. erw. Aufl., München 1959, S. 38-44.

15 Ebd., S. 46.

16 Vgl. ebd., S. 45, 47.

17 Daniel N. Stern, Die Lebenserfahrung des Säuglings (1986), Stuttgart 1992. 
nur wenige Tage alte Neugeborene auf das Aussehen von Gegenständen rückschließen können, deren Form sie zuvor nur ertastet hatten. So werde etwa ein Noppenschnuller aus einer Reihe anderer wiedererkannt, der zuvor im Mund bei verbundenen Augen intensiv bearbeitet wurde. Säuglinge nähmen ursprünglich abstrakte Repräsentationen wahr. Dabei handele es sich nicht "um Bilder, Töne, haptische Eindrücke und benennbare Objekte, sondern vielmehr um Formen,

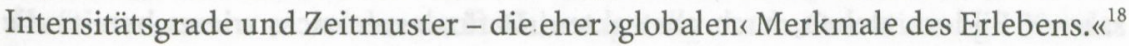
In Erweiterung seines Ansatzes verwies Stern, wie zuvor bereits Werner, auf die spezifische Qualität dieses Erlebens, die dazu neige, Wahrnehmungsqualitäten in Gefühlsqualitäten zu übersetzen. Stern kennzeichnete sie entsprechend als "Vitalitätsaffekte». Sie ließen sich am ehesten mit dynamischen, kinetischen Begriffen wie "aufwallend ", "verblassend ", "explosionsartig", "abklingend ", "berstend «, »sich anziehend" usw. charakterisieren. ${ }^{19}$ Das Vermögen, zwischen ihnen zu differenzieren, sei angeboren. Es zeichne sich durch eine »kraftvolle Zielstrebigkeit« aus, um zur Sicherung sozialer Interaktionen bzw. der Kommunikation zu dienen. ${ }^{20}$ Bereits von Stern selbst wurde es in einen unmittelbaren Zusammenhang zur Bild- bzw. Kunstwahrnehmung gebracht. So sah Stern im Stil, das heißt in der Weise wie der Maler die Formen handhabt, ein Pendant zum spontanen Verhalten im Bereich der Vitalitätsaffekte: „Die Übersetzung von der Wahrnehmung ins Gefühl erfordert also im Falle des künstlerischen Stils die Umwandlung rwahrheitsgetreuer Wahrnehmungen (Farbharmonien, Linienführungen usw.) in virtuelle Formen des Gefühls, zum Beispiel des Gefühls der Stille. ${ }^{21}$ Der Unterschied im Erfassen der Vitalitätsaffekte im sozialen Verhalten und in der Kunst liege, so Stern in Anlehnung an die Cassirer-Forscherin Susanne K. Langer, in der Kontemplation und damit Bewusstheit, die die Kunsterfahrung eröffne, die im »normalen « Leben durch »Verstrickungen in kontingente Umstände« meist unmöglich sei. ${ }^{22}$

Die von Stern herausgestellten Zusammenhänge treffen sich in bemerkenswerter Weise mit den Spiegelneuronenforschungen seit 1996. Sie machen deutlich, dass, wenn eine Aktion eines Anderen beobachtet wird, im Hirn dieselben Regionen aktiviert werden, als ob sie von ihm selbst ausgeführt würde. Diese Spiegelung des Verhaltens wird für das Verstehen des Anderen und für das soziale Verhalten des Menschen als zentral angesehen. Im Anschluss an die Untersuchungsergebnisse von Stern stellte sich weiterführend die Frage, ob bei der nachahmenden Beobachtung zwischen dem, was mit einer Handlung beabsichtigt wird (»goal and intention«), und dem, wie sie ausgeführt wird (»vi-

18 Ebd., S. 74-103, Zitat S. 80.

19 Ebd., S. 83.

20 Ebd., S. 49.

21 Ebd., S. 225-230, Zitat S. 227 f. Vgl. etwa oben die Beschreibungen zu Cézanne.

22 Ebd., S. 228. 
tality form «), unterschieden wird. Die direkte Zusammenarbeit Sterns mit einer Forschergruppe um einen der ursprünglichen Entdecker der Spiegelneuronen, Giacomo Rizzolatti, bestätigte diese Annahme. ${ }^{23}$ Ein weiteres Forschungsmitglied dieser Gruppe, Vittorio Gallese, stellte bereits 2007 gemeinsam mit dem amerikanischen Kunsthistoriker David Freedberg konkrete Bezüge zur Kunst her. Sie zeigten auf, dass wir auch dann nachahmend tätig seien, wenn wir vor starren Bildern stehen, auch abstrakten. Dafür lassen sich, wie es bereits die Entwicklungspsychologen betont hatten, neben figurativen insbesondere formale Qualitäten der Werke verantwortlich machen:

»With abstract paintings such as those by Jackson Pollock, viewers often experience a sense of bodily involvement with the movements that are implied by the physical traces - in brushmarks or paint drippings - of the creative actions of the producer of the work. This also applies to the cut canvases of Lucio Fontana, where sight of the slashed painting invites a sense of empathetic movement that seems to coincide with the gesture felt to have produced the tear. $\mathrm{i}^{24}$

Weiterführend beschrieb Gallese 2012 in einem Aufsatz mit Cinzia di Dio, dass diese Einfühlung in Bilder (»empathic feeling") sich als ein unmittelbar ablaufender Prozess (»automatic emotional response«) beschreiben lasse. Die Weise, wie ein Künstler etwa den Himmel in einem Landschaftsbild gestaltet, werde nicht nur nachvollzogen, sondern zugleich als lebendig bewegt bewertet. Damit werde das Kunstwerk zum Mediator zwischen Künstler und Betrachter: „Observers are likely able to appreciate the violent nature of the artwork because those brush strokes feature the movements they resonate with by means of the mirror mechanism. ${ }^{25}$

Für die Frage, inwiefern Bilder als historische Akteure verstanden werden können, erweisen sich im Anschluss an Werner die letzten beiden Ansätze als weitreichend. Stern benutzte für den Zusammenhang, den er zwischen der Wahrnehmung von Welt und Kunst feststellte, den Begriff des "Pendants «. Gallese verweist mit seinen Forschungen zur Perzeption von Anderen und der Kunst auf den Begriff der "Nachahmung ". Beide heben dabei zudem auf "Gefühlsqualitäten " (Stern) bzw. "Empathie" (Gallese) ab, die bei der Wahrnehmung aktiviert werden. Die Wahrnehmung wird - was sich als bemerkenswert herausstellt - nicht von den Inhalten oder angenommenen Bedeutungen angeregt, sondern von abstrakten Elementen: dem Heben der Arme beim Greifen

23 Vgl. Guiseppe di Cesare u.a., The neural correlates of "vitality form « recognition: an fMRI study, in: Social Cognitive and Affective Neuroscience 9 (2014), S. 951 -960.

24 David Freedberg / Vittorio Gallese, Motion, emotion and empathy in esthetic experience, in: Trends in Cognitive Sciences 11 (2007), S. 197 -203, Zitat S. 197.

25 Vittorio Gallese / Cinzia Di Dio, Neuroesthetics: The Body in Esthetic Experience, in: Vilayanur S. Ramachandran (Hg.), Encyclopedia of Human Behavior, Bd. 2: E-O, 2. Aufl., London u. a. 2012, S. $687-693$, S. 691. 
etwa nach einer Banane, dem Nachvollziehen von Pinselstrichen auf einer Leinwand (Gallese) bzw. den verschiedenen Intensitätsgraden, Formen und Zeitmustern, die zur Unterscheidung von Schnullern, Lauten der Mutter etc. wahrgenommen werden (Stern). Verstehen und Kommunizieren erfolgt hier ohne sprachliche Artikulation. Beides beruht auf den von (virtuellen) Bewegungsgestalten ausgelösten Empfindungen und dem Abgleich mit eigenen Bedürfnissen, seien es solche nach Nahrung oder nach Orientierung. Für das Verständnis von Bildern als historischen Akteuren ist wesentlich, dass beide Ansätze - wie in der formalen Ästhetik - davon ausgehen, dass nicht nur zwischen den Wahrnehmungs- und Handlungs- bzw. Kommunikationsweisen, sondern auch zwischen den Gestaltungs- und Anschauungsweisen eine Analogie bestehe. Sie funktionieren nach den gleichen Regeln, die auf abstrakten, affektivemotionalen und daher handlungsrelevanten Prinzipien beruhen. Insbesondere Kunst vermöge auf diese Vorgänge aufmerksam zu machen, während andere Bildwerke, so lässt sich anschließen, wie etwa solche der Werbung und Propaganda weniger diesen Zweck verfolgen, sondern dementgegen auf eine reine, möglichst unkritische Nachfolge bzw. den Kauf von etwas abzielen.

\subsection{Symbol- und Verkörperungstheorien}

Dass auch Ernst Cassirer von körpereigenen Prozessen ausging, die für den Menschen in seiner Hinwendung zur Welt und darüber hinaus für deren Auslegung als symbolisch bedeutsam zentral sind, entwickelte er in den drei Bänden zur »Philosophie der symbolischen Formen« zwischen 1923 und 1929. Demnach nehme der Mensch die Welt, wie Cassirer es zusammenfassend und letztlich in Übereinstimmung mit Heinz Werner im dritten Band festhielt, nicht sachlich auf, sondern affektiv-emotional gefärbt. ${ }^{26}$ Die Wahrnehmungsweise von Welt beruhe auf einer »starken triebhaften Unterschicht $«{ }^{27}$ Entsprechend bezeichnete Cassirer diese Form der Weltzuwendung als »Ausdrucks-Wahrnehmung«. Ein »Absehen« sei von dieser ursprünglichen Zugangsweise zur Welt nicht möglich: »[K]eine noch soweit getriebene Abstraktion vermag diese Schicht als solche zu beseitigen und auszulöschen «. ${ }^{28}$ Auf die konkrete Frage danach, was und wie wir wahrnehmen, verwies Cassirer auf Bewegungsgestalten und Raumformen, die als lebendig aufgegriffen werden:

26 Ernst Cassirer, Philosophie der symbolischen Formen, Bd. 3: Die Phänomenologie der Erkenntnis (1929), Darmstadt 1964, S. 86.

27 Ebd., S. 78.

28 Ebd., S. 86. 
»In Wahrheit bedeutet, innerhalb dieses Horizontes, die Ausdrucks-Wahrnehmung gegenüber der Ding-Wahrnehmung nicht nur das psychologisch-Frühere [...]. Sie hat ihre spezifische Form, ihre eigene ,Wesenheit , die sich nicht durch Kategorien, die für die Bestimmung ganz anderer Seins- und Sinnregionen gelten, beschreiben, geschweige durch sie ersetzen läßt. [...] im Spiegel der Sprache [...] läßt sich zumeist noch unmittelbar erkennen, wie alle Wahrnehmung eines ,Objektiven ursprünglich von der Erfassung und Unterscheidung gewisser 'physiognomischer Charaktere ausgeht, und wie sie mit diesen gleichsam gesättigt bleibt. Die sprachliche Bezeichnung einer bestimmten Bewegung etwa birgt fast durchweg dieses Moment in sich: statt die Form der Bewegung als solche, als Form eines objektiven raum-zeitlichen Geschehens, zu beschreiben, wird vielmehr der Zustand genannt und sprachlich fixiert, von dem die betreffende Bewegung der Ausdruck ist. >Raschheit , ,Langsamkeit und zur Not noch ,Eckigkeit, so heißt es bei Klages [...], mögen rein mathematisch verstanden werden;

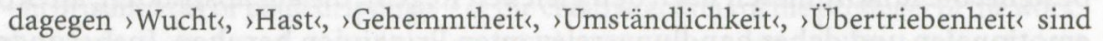
ebenso sehr Namen für Lebenszustände wie für Bewegungsweisen und beschreiben in Wahrheit diese durch Angabe ihrer Charaktere. Wer Bewegungsgestalten und Raumformen kennzeichnen will, findet sich unversehens in eine Kennzeichnung von Seeleneigenschaften verstrickt, weil Formen und Bewegungen als Seelenerscheinungen erlebt worden sind, ehe sie aus dem Gesichtspunkt der Gegenständlichkeit vom Verstande beurteilt werden, und weil die sprachliche Verlautbarung der Sachbegriffe nur durch Vermittlung von Eindruckserlebnissen stattfindet. ${ }^{29}$

Entsprechend charakterisierte Cassirer diese ursprüngliche Form des Wahrnehmens als eine, die durch ein Erleben und Erleiden gekennzeichnet sei. Dasjenige, was erfasst werde, erhalte dadurch einen Ausdruck: "Ausdruck ist zunächst nichts anderes als ein Erleiden; ist weit mehr ein Ergriffenwerden als ein Ergreifen ${ }^{30}{ }^{30}$ Diese Beschreibungen weisen auf zwei Seiten der ursprünglichen Wahrnehmungsweise hin: zum einen auf das unmittelbare Reagieren auf äußere Reize (Bewegungsgestalten und Raumformen) und zum anderen auf deren affektiv-emotionale Aufnahme (Ausdruck). Dasjenige, was wahrgenommen wird, sind abstrakte Formen, und die Weise, wie wahrgenommen wird, ist nicht-diskursiv. Dennoch - und hierin liegt die eigentliche Bedeutung dieses Formverständnisses - vermittele sich über sie bereits ein erster Sinn. Er liege darin, dass die je spezifisch als dynamisch wahrgenommenen abstrakten Elemente über das Affekt- und Willensleben bzw. über die Bild- und Tatkraft affektiv-emotional ausgelegt werden. Nach Cassirer liegen die Voraussetzungen für jedes Handeln demnach in der affektiv-emotionalen Auslegung, die einen ersten symbolischen Sinn erzeugt, an dem sich der Einzelne ausrichtet: "So beruht das geschichtliche Bewußtsein auf einem Ineinander und einer Wechselwirkung von Tatkraft und Bildkraft: auf der Klarheit und Sicherheit, mit der das Ich imstande ist, ein zukünftiges Sein im Bilde vor sich hinzustellen und alles

29 Ebd., S. 94. Kursive Wörter im Original gesperrt.

30 Ebd., S. 88. Kursives Wort im Original gesperrt. 
einzelne Tun auf dieses Bild zu richten. ${ }^{31}$ Die ersten Bilder (»Symbole $«$ ), die uns dieses »Tun« des Menschen vermittelt, seien die einer lebendigen Ausdruckwelt (»mythisches Bewusstsein «). ${ }^{32}$ So galt auch für Cassirer, wie es ebenso Werner und später Stern herausstellten: "Das ,Verstehen von Ausdruck ist wesentlich früher als das ,Wissen von Dingen.$"{ }^{33}$ Weiterführend gelte, dass - auch wenn die Logik der später gewonnenen Ding- und Kausalbegriffe uns veranlasse, alle Brücken zur reinen Ausdruckwelt abzubrechen - die ursprüngliche affektivemotionale Wahrnehmungsweise dennoch nicht verloren gehe. ${ }^{34}$ Die zentrale Funktion, die sie für das Handeln übernimmt, mag hierfür eine Erklärung bieten, die Cassirer selbst nicht weiter ausgearbeitet hat.

Erst 1944 mit der im amerikanischen Exil veröffentlichten Schrift »An Essay on Man " griff Cassirer die Thematik nochmals auf und betonte, was für die hier verfolgte Fragestellung wichtig ist, dass diese ursprüngliche, lebendige, von uns nicht länger anerkannte Weltauffassung in der Kunst anschaubar werde. ${ }^{35}$ Ebenso wie es später Langer und Krois bekräftigten, gehe mit der Übertragung in die Kunst jedoch die Handlungsrelevanz verloren. Es sei allein das ästhetische Erlebnis der »lebendigen Formen«, wie sie die Bilder vermitteln, das als solches bedeutsam werde. So führte Cassirer hierzu aus, dass für den Künstler die Macht der Leidenschaft, die in der ursprünglichen affektiven Weltauffassung liege, »zu einer bildenden, formgebenden Kraft « werde, wobei etwa der tragische Dichter "nicht Sklave, sondern Herr seiner Gefühle» sei. Mit dem Werk erfahre der ursprüngliche emotionale Gehalt einen Gestaltwandel, der auch für den Rezipienten bedeutsam wird. In der Welt der »reinen Sinnesformen « werde den Leidenschaften ihre dingliche Bürde genommen. Sie würden von der Kunst in Handlungen, in Motion statt Emotion, in einen dynamischen Prozess inneren Lebens, der den Betrachter / Zuhörer bewegt, verwandelt. ${ }^{36}$ Exemplarisch verdeutlichte Cassirer diesen Perspektivenwechsel, indem er die Erfahrungen mit einer natürlich schönen Landschaft »mit den Augen eines Künstlers « beschrieb:

"[I]ch fange an ein Bild von ihr [der Landschaft, M.S.] zu formen. Damit habe ich ein neues Terrain betreten, das Feld nicht der lebendigen Dinge, sondern der lebendigen Formen. Nicht mehr in der unmittelbaren Wirklichkeit der Dinge stehend, bewege ich mich nun im Rhythmus der räumlichen Formen, in der Harmonie und im Kontrast der

31 Vgl. grundlegend zur Bild- und Tatkraft ebd., S. 189-221, Zitat S. 212. Kursive Wörter im Original gesperrt.

32 Ebd., S. 71-74.

33 Ebd., S. 73 f. Vgl. hierzu Werner, S. 154-173 (\$ 35); ergänzend zu Stern, S. 48 f.; Ausarbeitung dazu S. 231-258.

34 Cassirer, Phänomenologie der Erkenntnis, S. 99 f.

35 Ders., An Essay on Man (1944); dt.: Versuch über den Menschen. Einführung in eine Philosophie der Kultur, 2., verb. Aufl., Hamburg 2007.

36 Ebd., S. 212-234, Zitat S. 229. 
Farben, im Gleichgewicht von Licht und Schatten. Der Eintritt in die Dynamik der Form begründet das ästhetische Erlebnis. «"

Weiterführend sprach Cassirer von einer »Intensivierung von Wirklichkeit«, die über die Gestaltung und Wahrnehmung von Kunst erfolge. ${ }^{38}$ Doch genau darin, ebenso wie später bei Langer und Krois, lassen sich Widersprüche zu seinem ursprünglich entwickelten Ansatz aufzeigen. Denn ist nicht schon die Intensivierung von etwas eine Steigerung, die einer Ansicht gleich eine spezifische Sichtweise / Meinung von etwas vermittelt? Wird dem zugestimmt, dann ist es die Sichtweise / Auslegung des Künstlers, die mit der Gestaltung in das Bild gelegt und vom Betrachter verstanden werden kann. Für die Wahrnehmung eines Landschaftsbildes mag die Steigerung / Deutung des Künstlers noch als ästhetischer Genuss im Sinn einer Intensivierung der Wirklichkeitserfahrung aufgenommen werden, wählt der Künstler jedoch andere Themen wie etwa politisch oder werbetechnisch relevante, ändert sich die Situation. Auch in diesen Fällen lässt sich von einer Intensivierung sprechen, die die gewählten Themen in einem bestimmten Licht erscheinen lässt, die über die Steigerung der Empfindungen dann jedoch handlungsrelevant werden können, indem sie das Begehren zum Erwerb oder zur Nachahmung von Ideen wecken.

In der Nachfolge von Cassirer hat vor allem die amerikanische Philosophin Susanne K. Langer bereits in ihrem 1942 erschienenen Buch »Philosophy in a New Key« betont und in späteren Schriften vertieft: »Alle unsere Anzeichen und Symbole sind jedoch sinnlicher und emotionaler Erfahrung entnommen und tragen den Stempel ihres Ursprungs. ${ }^{39}$ Im Anschluss an Cassirer sei dieser Zusammenhang für die Kunst und deren Rezeption wesentlich. Statt jedoch die "Intensivierung und Erhellung " der Wirklichkeit ${ }^{40}$ in den Vordergrund zu stellen, betonte Langer die Bedeutsamkeit der Gefühlsebene selbst und damit deren nicht-diskursiven Charakter. So vermerkte sie, dass »der Gefühlsinhalt des Werkes [Hervorhebung M.S.] [...] vorrational, wesentlicher und lebendiger, von der Art des Lebensrhythmus [ist], den wir mit allen wachsenden, hungernden, sich regenden und furchtempfindenden Geschöpfen teilen: er betrifft letzte Wirklichkeiten, die zentralen Fakten unseres kurzen, bewussten Daseins. ${ }^{41}$ Ihn zu verstehen, setze eine Vertrautheit mit der »impliziten " (und nicht diskursiven oder präsentativen) Bedeutung der Werke voraus, die eigener, "nichtdiskursiver« Formen des Begreifens bedürfe. ${ }^{42}$

37 Ebd., S. 233 f.

38 Ebd., S. 221.

39 Susanne K. Langer, Philosophy in a New Key (1942); dt.: Philosophie auf neuem Wege. Das Symbol im Denken, im Ritus und in der Kunst, 4. Aufl., Berlin 1965, S. 241 - 260, Zitat S. 254.

40 Cassirer, Versuch über den Menschen, S. 228.

41 Langer, S. 278.

42 Ebd., S. 256-260. 
Den nicht-diskursiven Formen des Begreifens entsprechen - wie es indirekt bereits Cassirer herausarbeitete und auch von Werner und weiterführend von Stern und Gallese betont wurde - formal-abstrakte Gestaltungsweisen. Letztere gründen nach Langer in der Musik in der "tonalen dynamischen Form « und in der Malerei, Bildhauerei und Dichtung im »Spiel der Linien, Massen, Farben und Stoffe « - wobei der Gehalt des künstlerischen Ausdrucks selbst, wie ihn Langer in dieser frühen Schrift noch als Vermutung nahe legte und in »Feeling and Form« 1953 zum Thema machte, in allen Künsten der gleiche wie in der Musik sei. ${ }^{43}$ Der Gehalt sei »das mit Worten nicht sagbare, und doch nicht unausdrückliche Prinzip der lebendigen Erfahrung, die innere Bewegungsform des empfindenden, seines Lebens bewussten Daseins. ${ }^{44}$ Entsprechend definierte Langer die Kunst als »the creation of forms symbolic of human feeling $« .{ }^{45}$ Die konkrete symbolbildende Kraft liege darin, dass über die Spannungen und Entspannungen der bildnerischen Mittel (»tensions and resolutions ${ }^{46}$ ) ein virtuelles Bild organischen Lebens entstehe. ${ }^{47}$ Sie beruhe auf der engen Beziehung zwischen organischen [leiblichen, M.S.] bzw. mentalen Prozessen (»vital forms«) und künstlerischen Formen (»artistic forms«). Zwischen ihnen müsse, wie es auch Wiesing in Auseinandersetzung mit der formalen Ästhetik aufzeigt, eine Analogie bestehen. Darauf baut die (Bild-)Akt-Theorie Langers auf. ${ }^{48}$ So wie bereits Cassirer ansetzte, betonte Langer, dass die Elemente der Kunst erst im Zusammenspiel symbolisch (gefühlsmäßig) bedeutsam werden. Im logischen bzw. dialektischen Muster (»tension and resolution«) der möglichen Beziehungen ("potential acts") forme sich der Sinn der Erscheinung als "lebendige Form « bzw. »illusion of bodily existence «. ${ }^{49}$ Die Vergleichbarkeit von biologischen und künstlerischen Akten liege in dem vitalen Vorgang der Über- und Unterordnung von einzelnen Akten. ${ }^{50}$ Funktional betrachtet besteht darin die

43 Dies., Feeling and Form. A Theory of Art Developed from Philosophy in a New Key (1953), 4. Aufl., London 1967, S. 103, 369, 372.

44 Dies., Philosophie auf neuem Wege, S. 252.

45 Dies., Feeling and Form, S. 40.

46 Vgl. hierzu grundlegend Langers Ausarbeitung in dem 1967 und 1972 vorgelegten Doppelband: dies., Mind: An Essay on Human Feeling, Bd. 1 (1967), 4. Aufl., Baltimore / London 1985, Bd. 2, Baltimore / London 1972. Vgl. hier Bd. 1, S. 206 f.

47 Dies., Feeling and Form, S. 207; vgl. ergänzend S. 47-59, 372.

48 Dies., Mind, Bd. 1, S. 199-253, bes.. S. 200 f., 211.

49 Ebd., S. 206 f.

50 Ebd., S. 261-268. Diese Annahme wurde von Langer dahingehend ausgeweitet, dass sie bereits auf der Ebene der molekularen Interaktionen, die von physikalischen und chemischen Prozessen gesteuert werden, eine Vergleichbarkeit bis hoch zu organischen und psychischen und weiterführend künstlerischen Prozessen annimmt. Eine These, mit der sie unmittelbar an ihren Lehrer und Freund Alfred North Whitehead anschloss, dem sie entsprechend den Doppelband widmete. Vgl. zu Whitehead, Lachmann, S. $122 \mathrm{ff}$. Den Nachweis 
Möglichkeit der Übertragung von einer auf die andere Ebene. So könne das imaginative und gedankliche Vermögen beim Menschen die Aufgabe übernehmen, dem alltäglichen Strom von Spannungen und Entspannungen zu begegnen und ihn zu bannen. ${ }^{51}$ Der illusionäre Charakter der Kunst erlaube es dem Betrachter darüber hinaus, ihr frei von praktischen Zwecken zu begegnen. Er ermögliche eine Distanz, die im Ritual und in der Unterhaltung verloren gehe, indem Letztere durch größtmögliche Nähe und einem Glauben-Machen eine Delusion (Eins-Sein) erzeugen. ${ }^{52}$

Doch schließt die erkennbare Zweckorientierung im Ritual und in der Unterhaltung tatsächlich aus, dass sie sich auf von Gefühlen geprägte Erfahrungen des Menschen in der Welt beziehen, wie Langer vermutet? Diese später geäuBerte Annahme, ${ }^{53}$ die ihr letztlich erlaubte, an der klassischen ästhetischen Theorie festzuhalten, widerspricht jedoch ihrem eigenen Ansatz. Demzufolge könne der stete Strom der "tensions and resolutions" in bildnerische Mittel umgesetzt werden, so dass wir über die Gestaltungen - seien es solche der Kunst und des Designs oder der Werbung und der Propaganda - ein lebendiges, virtuelles Bild einzelner Aspekte davon gewinnen können. Berechtigt scheint die These Langers dagegen mit Bezug auf die Praktiken der Delusion, die uns unter Umgehung unseres bewussten Denkens ihre Ansichten vermitteln können, ohne auf merklichen Widerstand zu stoßen. Die Zweifel an Langers grundsätzlicher Unterscheidung zwischen Kunst und Ritual / Unterhaltung werden zudem durch ihre eigenen Schlussfolgerungen bestärkt, die sie in ihrem zweibändigen Spätwerk »Mind. An Essay on Human Feeling « (1967/72) vorstellte. Denn wenn über die Form (»external forms") eine Objektivierung der Gefühle stattfindet und zugleich mit Bezug zum Inhalt eine Subjektivierung der Natur (»internal forms «) einhergeht, ${ }^{54}$ so liegt es im Ermessen des Künstlers / Gestalters, was von ihm wie subjektiviert wird. Sein Vermögen besteht dann nicht nur darin, »forms symbolic of human feeling " zu erschaffen, sondern über die "quality of expression ${ }^{55}$ und die Wahl der Themen eigene Zwecke zu verfolgen. Vor diesem Hintergrund mit Langer von einem "presentational symbolism « zu sprechen, jedoch nicht nur im Sinn der Präsentation von Gefühlen, sondern darüber hinaus von Ansichten, gilt es hier in Erweiterung vorzuschlagen.

Das (Bild-)Aktverständnis, wie es Langer in ihren Schriften entwickelte, deckt sich in vielerlei Hinsicht mit dem des Cassirer-Experten John M. Krois. So ging

führte Langer, indem sie sich ergänzend auf Forschungsergebnisse aus der Psychologie und

Entwicklungspsychologie, Physiologie, Neurologie, Biologie und Zoologie stützte.

51 Langer, Feeling and Form, S. 371-375.

52 Ebd., S. 319 f.

53 Dies., Mind, Bd. 1, S. 127 f.

54 Ebd., S. 86 f.; vgl. ergänzend die Schlussbetrachtung in dies., Mind, Bd. 2, S. 342.

55 Dies., Mind, Bd. 1, S. 127. 
auch er davon aus, dass es dem Menschen möglich ist, über die Künste an den Fluss des Lebens anzuschließen, der von Gefühlsbewegungen geprägt sei. Doch mit Langers Forschungen hatte sich Krois nur am Rande beschäftigt, ${ }^{56}$ stattdessen knüpfte er ebenfalls unmittelbar an Cassirer an. Bereits in der Schrift zu Cassirers Geschichtsauffassung 1987 und vertiefend in einer Reihe von Aufsätzen seit $1998^{57}$ untersuchte Krois die Voraussetzungen für diese Annahme. Wie bereits Langer betonte, sei es die Kunst, die eine Objektivierung dieser Gefühlswelt erlaube: "In art, a medium permits giving expressive meaning an objective form. ${ }^{58}$ Die expressive Bedeutung liege schon immer vor und sei grundsätzlich nicht-diskursiver Natur. ${ }^{59}$ Sie betreffe die Wahrnehmung in ihrer ganzen körperlichen Gebundenheit: „Expressive meaning is not a product of culture; it characterizes the first stages of perception and bodily awareness [...]. This is the prototype of all symbolic relations. ${ }^{60}$ Das ausdrucksmäßige Verstehen, wie es Cassirer einführte, kann daher, so lässt sich an die Ausarbeitung von Krois an früherer Stelle anschließen, als Funktion aller höheren symbolischen Formen (mit symbolischer Prägnanz) verstanden werden: Sie sei »the logical structure of experience ${ }^{61}$ - eine Schlussfolgerung, die auch Langer gezogen und der Ausarbeitung ihrer (Bild-)Akt-Theorie zu Grunde gelegt hatte. ${ }^{62}$ Krois wertete den Ansatz Cassirers so aus, dass er nicht von einer formalen Relation von Bedeutungszusammenhängen ausging, einer auf Vergleich beruhenden Ordnung nach Klassen und logischen Schlüssen, sondern von einer »erzeugenden Relation«, die die Grundlage aller Ordnungszusammenhänge bilde. ${ }^{63}$ In der Kunst zeige sie sich in einer Weise, dass die expressive Ausdeutung (»die erzeugende Relation «) für uns erkennbar und diskutierbar werde. ${ }^{64}$

Die Grundlagen dieser Annahme zu klären, stellte sich Krois als Aufgabe, wie die nachfolgenden Aufsätze und Aktivitäten zeigen. ${ }^{65}$ Dabei blieb für Krois die

56 Vgl. John M. Krois, Experiencing Emotion in Depictions. Being Moved without Motion? (2010), in: ders., Bildkörper und Körperschema. Schriften zur Verkörperungstheorie ikonischer Formen, hrsg. von Horst Bredekamp / Marion Lauschke, Berlin 2011, S. 232-251, hier S. $247 \mathrm{f}$.

57 Sie wurden 2011 von Horst Bredekamp und Marion Lauschke herausgegeben; vgl. vorige Anm.

58 John M. Krois, Cassirer: Symbolic Forms and History, New Haven / London 1987, S. 132.

59 Ebd., S. 59.

60 Ebd., S. 57; vgl. ferner S. 85 f.

61 Ebd., S. 47.

62 Langer, Mind, Bd. 1, S. 272-299, 324.

63 Krois, Cassirer, S. 44-62, Zitat S. 45.

64 Ebd., S. 133.

65 So gründete Krois gemeinsam mit dem Kunsthistoriker Horst Bredekamp 2008 das Forschungskolleg "Bildakt und Verkörperung" an der Humboldt-Universität in Berlin. Vor diesem Hintergrund kann Krois auch als ein Mitideengeber des seit 2012 laufenden Exzellenzclusters »Bild Wissen Gestaltung " angesehen werden, das von Bredekamp und dem 
Frage, wie in der Begegnung mit Kunst Gefühle angesprochen werden, lange unbeantwortet. ${ }^{66}$ Erst spät, konkret in Auseinandersetzung mit dem Enaktivismus seit 2010, ${ }^{67}$ eröffnete sich ihm, dass sich Empfindungen nicht nur an die Willensbildung knüpfen lassen und damit an die von ihr in einem Abwägungsprozess verfolgten Wünsche und Zwecke (»Evaluationsprozess ${ }^{68}{ }^{6}$ ), sondern an den Wahrnehmungsprozess selbst. So gewann die von Krois in mehreren Aufsätzen angenäherte Verkörperungstheorie vor allem in seinem letzten Lebensjahr an Kontur. Eine zusammenhängende Ausarbeitung war ihm nicht mehr möglich.

Im Nachfolgenden werden die Grundzüge der These mit Bezug auf verschiedene Aufsätze aufgezeigt. Den Ausgangspunkt bildete für Krois die Annahme, dass sowohl das Bild als auch der Körper (bzw. die Wahrnehmung des Menschen, aber auch von Tieren und Robotern) auf vergleichbaren Prinzipien bzw. Schemata aufbauen. Zwischen den Bildschemata und Körperschemata besteht eine Analogie: »Bei allen werden die Körperschemata aus den gleichen Bildschemata aufgebaut. Diese Bildschemata sind dynamische, nicht optische Formen «. Diese bewusst zu erleben und zu fühlen (als "Qualitäten « ${ }^{69}$ über die "Ausdruckswahrnehmung ${ }^{70}$ ), zeichne den Menschen im Gegensatz zum Tier aus. Diese Annahme deckt sich, wie sich bereits zeigte, mit der von Langer, Werner, Stern und Gallese. So waren es auch für Krois unbewusste, senso-motorische bzw. körperliche (Propriozeption) und damit nicht-diskursive Prozesse (des Körperschemas), die sowohl als Grundlage für eine bewusste, lebendige Selbsterfahrung (des Körperbildes) als auch für aktive Formbildungs- und Wahrnehmungsprozesse von Bildern und Zeichnungen (des Bildkörpers) angesehen werden können. ${ }^{71}$ Sie ermöglichen eine räumliche Orientierung und

Kulturwissenschaftler Wolfgang Schäffner eingerichtet wurde: Vgl. http://www.kunst geschichte.hu-berlin.de/2012/07/exzellenzcluster-bild-wissen-gestaltung; https://www.inter disciplinary-laboratory.hu-berlin.de/de.

66 John M. Krois, Für Bilder braucht man keine Augen. Zur Verkörperungstheorie des Ikonischen (2006), in: ders., Bildkörper und Körperschema, S. 132-160, hier S. 160.

67 Ders., Experiencing Emotion in Depictions, S. $237 \mathrm{f}$.

68 Vgl. ders., Cassirer, S. 155, 167, 102-105.

69 Ders., Tastbilder. Zur Verkörperungstheorie ikonischer Formen (2010), in: ders., Bildkörper und Körperschema, S. 208 - 231, hier S. 231. Vgl. zum tieferen Verständnis der "Qualitäten" zudem seine Auseinandersetzung mit Charles S. Peirce: ders., Image Science and Embodiment or: Peirce as Image Scientist (2009), in: ders., Bildkörper und Körperschema, S. 194 209, bes. S. $202-209$.

70 Ders., Bildkörper und Körperschema (2011), in: ders., Bildkörper und Körperschema, S. $252-271$, hier S. 270. Krois stellt hier keinen unmittelbaren Bezug zu Cassirers Bestimmungen her, die er jedoch an anderer Stelle erörtert hat; vgl. ders., Für Bilder braucht man keine Augen, S. 142; ders., Synesthesia and the Theory of Signs (2007), in: ders., Bildkörper und Körperschema, S. 162-174, hier S. 171.

71 Ders., Tastbilder, S. 221-231, Zitat S. 231; ergänzend, ders., Bildkörper und Körperschema, S. $252-271$. 
können daher als grundlegend für emergente und damit nicht auf Repräsentation beruhende, intelligente Handlungen betrachtet werden. ${ }^{72}$ Demnach sind es in Anlehnung an die Verkörperungstheorie von Charles S. Peirce ("philosophy of embodiment « $^{73}$ ) die von ihm als »Qualitäten« (mit Peirce »icons«) bestimmten nicht-diskursiven, nicht optischen Formen, deren Veränderungen erfahren und ausgedeutet werden können. Doch Peirce sah dieses Ausdeuten nur als einen Prozess des Denkens, eine letztlich unbewusste Verhaltensweise (Handlung) an, die die Verhaltensänderung erklärt, ${ }^{74}$ während Krois im Anschluss an Cassirer hierfür die Ausdrucks-Wahrnehmung in Anschlag brachte, über die ein Ausdeuten der dynamischen, nicht-optischen Formen als gefühlte Qualitäten erfolge ${ }^{75}$ und die derart als unerlässlicher Anfang des Erkenntnisprozesses angesehen werden könne ${ }^{76}$ - ein Ansatz, der in vergleichbarer Weise sowohl von Cassirer und Langer als auch von Werner, Stern und Gallese verfolgt wurde.

Dennoch hielt Krois an verschiedenen Stellen - letztlich im Gegensatz zu seinen eigenen Aussagen - fest, dass, auch wenn im Bild dynamische, affektiv wirksame Aspekte liegen, diese unabhängig von den Intentionen des Künstlers und entsprechend auch der Interpretation des Betrachters seien. Daher sei das Bild keine Mitteilung und habe demzufolge auch keine Handlungsrelevanz: "The ursupatory character of pictorial objects - the fact that they possess affective meanings independently of the artists' intentions and the viewer's deliberate interpretations - results from the fact that like the viewer, they too embody dynamic affective image schemas. ${ }^{77}$

Obwohl Cassirer, Langer und Krois an der klassischen ästhetischen Theorie festhielten, unterstützen die von ihnen entwickelten und hier herausgearbeiteten Verkörperungstheorien in Übereinstimmung mit den Ergebnissen aus dem Bereich der Entwicklungspsychologie und der Neurowissenschaft die hier vertretene These, Bilder als historische Akteure aufzufassen. Auch sie gründeten ihre Forschungen auf der Annahme, dass körpereigene Prozesse nicht nur für

72 Ders., Tastbilder, S. 231 bzw. S. $227-231$.

73 Ders., Image Science and Embodiment, S. 198.

74 Vgl. hierzu Charles S. Peirces Vorlesungen von 1903 zum "Syllabus of Certain Topics of Logic«; dt.: Phänomen und Logik der Zeichen, hrsg. u. übers. von Helmut Pape, 2. Aufl., Frankfurt a. M. 1993, hier S. 54-58, 64 ff. Vgl. ergänzend den sehr aufschlussreichen Appendix II (S. 163 -171) zur Definition des Pragmatismus als "wofür das Denken geschieht" (S. 164), womit inhärent das Denken selbst als Zweck aufgezeigt wird (S. 171).

75 Krois, Image Science and Embodiment, S. 204-209.

76 Ders., Was sind und was wollen die Bilder? (2011), in: ders., Bildkörper und Körperschema, S. $290-306$, hier S. $303 \mathrm{f}$.

77 Ders., Experiencing Emotion in Depictions, S. 251; vgl. ferner ders., Bildkörper und Körperbilder, S. 269; ders., Enactivism and Embodiment in Picture Acts. The Chirality of Images, in: ders., Bildkörper und Körperschema, S. 272-289, hier S. 278; ders., Was sind und was wollen die Bilder, S. 306. 
die Sinnbildung dessen, was in der Welt wahrgenommen wird, sondern auch für die Wahrnehmung von Kunst bzw. - wie es hier zu präzisieren gilt - von Bildern im Allgemeinen, wesentlich sind. Demnach ist es auch für die kulturanthropologisch ausgerichtete Forschung ausschlaggebend, dass die Wahrnehmung im Allgemeinen und die von Kunst bzw. Bildern im Besonderen nicht neutral bzw. sachlich, sondern affektiv-emotional ist. Cassirer bezeichnete diese Form von Wahrnehmung als "Ausdrucks-Wahrnehmung", Langer verstand sie als sinnlich-emotional und Krois unterstellte ihr im Anschluss an Cassirer ein wexpressive meaning ". Diese Art der Wahrnehmung orientiert sich, ebenso wie es Werner, Stern und Gallese betonten, nicht an konkreten benennbaren Objekten und einer möglichen Bedeutung, die sie für uns haben können, sondern an abstrakten Formen. Übereinstimmend gingen auch sie davon aus, dass die gestalterischen Grundlagen in einer Analogie zu den Wahrnehmungsweisen stehen müssen. Letztere sind entsprechend der affektiv-emotionalen Verarbeitung des Bildnerischen leiblich gebundene Abläufe. Cassirer ordnete sie einer »stark triebhaften Unterschicht« zu, Langer dem Organischen und Krois dem Körper. Im Alltag erweist sich diese Analogie zwischen den Ebenen als grundlegend für die Handlungsrelevanz des Einzelnen; mit Bezug auf Bilder erfuhr diese von allen drei Forschern eine Differenzierung. Auch dort sei diese wirksam, ermögliche jedoch gerade mit Blick auf die Funktion von Kunst eine Durchlässigkeit zwischen den Ebenen, so dass vom Werk aus - nach Cassirer - die Weise, wie Welt wahrgenommen werde, erkennbar wird (»Intensivierung der Wirklichkeit«). Langer hob über die bewusste Erfahrung mit dem Werk auf das Gewahrwerden der Gefühlsebene im Menschen ab, während Krois die Unabhängigkeit und Eigentümlichkeit der Kunst betonte. Die Wirkung, die Kunst und Bilder hiernach haben, ist daher eine rein ästhetische.

Wird dementgegen davon ausgegangen, dass sowohl Werke der Kunst als auch Bilder im Allgemeinen stets Setzungen durch eine Person sind, dann können alle Bildformen für den Betrachter handlungsrelevant werden. Denn vor dem Hintergrund dieses Ansatzes verändert sich die Deutung dessen, was jeweils wahrgenommen wird. In dem Fall erhellt das Werk nicht nur die Vorstellungen vom Werk selbst (Krois), von der Wirklichkeit (Cassirer) oder der eigenen Gefühlsebene (Langer), sondern lässt sich als eine spezifische Deutung derjenigen Themen, die der Gestalter wählt, verstehen. Das Werk repräsentiert sein Bild der (Lebens-)Welt. Es ist seine (An-)Sicht von ihr. Als berechtigt erweist sich dieser Ansatz, wenn bedacht wird, dass Form und Inhalt nicht getrennt werden können. Über die affektiv-emotional geprägte Wahrnehmung der Form werden zugleich die jeweiligen Gestalten und Inhalte mit ausgelegt bzw. bewertet: eine Bewertung, die dann doch nicht diejenige des Betrachters ist, sondern bereits vorgegeben wurde. Mit dem Wahrnehmen des Bildes versteht damit der Betrachter das, was ihm ein Anderer "sagen " will. Daran anschließend 
bietet sich ihm die Möglichkeit, sich dazu zu stellen bzw. darauf zu reagieren, je nachdem affirmierend oder kritisch.

Vor dem Hintergrund der Frage, wie sich dieser Ansatz für die Analyse von Bildern als historischen Akteuren operationalisieren lässt, werden die Perspektiven des Ansatzes erkennbar. Sie liegen darin, dass sich die über die Form angeregten affektiv-emotionalen Anteile nur als Impulse beschreiben lassen, die in einem Abstimmungsprozess mit dem Inhalt und in Bezug auf die je eigenen gesellschaftlichen und kulturellen Voraussetzungen zu einem konkreten Tun veranlassen können. Über die Darstellungsweise werden daher nur wertebildende Prozesse angeregt, die erst über den Abgleich mit kulturell geprägten Vorwissen und Ansichten als handlungsrelevant angesehen werden können.

\section{Fazit: Das Bild als historischer Faktor}

Wie lassen sich Bilder als historische Akteure greifen? Die Untersuchung sollte zeigen, dass die Unterscheidung zwischen ästhetischen bzw. aisthetischen Bilderfahrungen, die als leiblich gebunden verstanden werden können, und rezeptionsgeschichtlichen Auslegungen von Bildern, in denen der kulturelle Kontext und das Vorwissen zur Anwendung kommen, sich nicht ausschließen müssen. Im Gegenteil, mit der Auswertung der hier vorgestellten kulturanthropologischen und der ergänzenden entwicklungspsychologischen und neurowissenschaftlichen Forschungen seit Beginn des letzten Jahrhunderts eröffnet sich, dass beide Teile für das Verständnis von Bildern als historischen Akteuren wesentlich sind. Als Grundlage dafür ist anzuerkennen, dass aus einer rein sachlich-neutralen Auflistung der Bildinhalte und der Analyse ihrer Bedeutungen keine Relevanz für Zukünftiges abgeleitet werden kann. Genau besehen schließen Verkörperungstheorien die Möglichkeit, neutral zu urteilen, sogar aus. Sowohl die Erfahrung des Bildes als ästhetischen Objekts als auch diejenige, die es als eine Ansicht im Sinn einer Meinung über etwas zu verstehen sucht, hängen davon $\mathrm{ab}$, die als stimulierend angenommene Wirkung der Impulse, wie sie der Bildgestalter über die angenommene, bestehende Analogie zwischen den Gestaltungs- und Anschauungsweisen dem Betrachter zu vermitteln vermag, als entscheidenden Faktor für wertebildende Prozesse anzunehmen. Für den Ansatz, Bilder selbst als historische Akteure zu bestimmen, schließt es sich jedoch aus, sie nur als eine Gestaltung von etwas (Ansicht / Thema) zu diskutieren (ästhetische Theorie / historische Quellenforschung). Der Einbezug des Künstlers / Gestalters als mit dem Bild Sprechenden erweist sich vor diesem Hintergrund als unerlässlich. Denn nur wenn das Bild als seine Ansicht / Meinung über etwas ernst genommen wird und entsprechend eine Antwort des 
Betrachters einzufordern vermag, erweist sich die Bilderfahrung, vergleichbar sprachlichen Äußerungen, als historisch relevant.

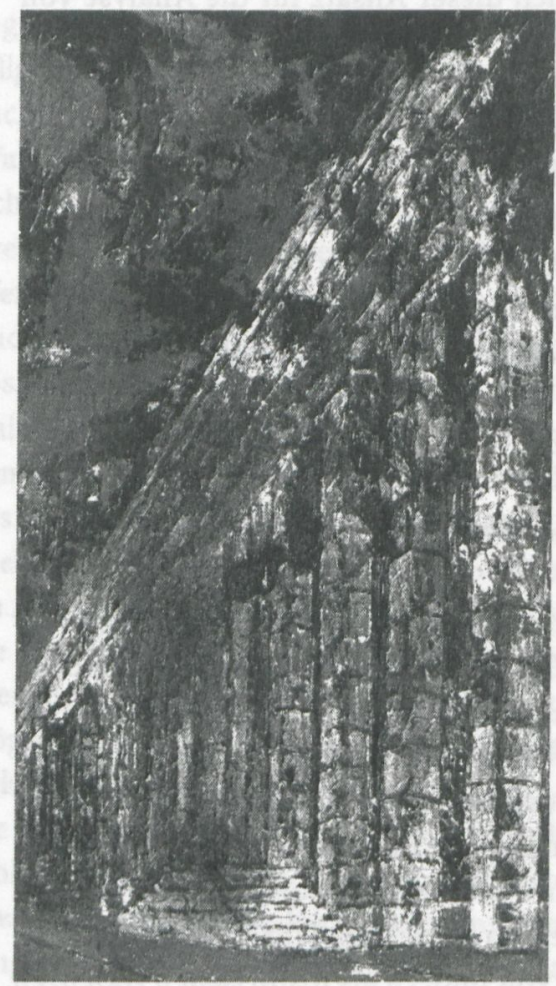

Abb. 2: Anselm Kiefer, «Die Treppe«, 1982/83, 330 x 185 cm, Öl, Emulsion, Stroh auf Fotografie auf Leinwand, Kunstmuseum Bonn. Aus: Martina Sauer, Faszination - Schrecken. Zur Handlungsrelevanz ästhetischer Erfahrung anhand Anselm Kiefers Deutschlandbilder, in: ART-Dok, 05.03.2012 (vgl. Anm. 78), S. 150 (Abb. 12).

Abschließend soll nochmals ein konkretes Beispiel diesen Zusammenhang anschaulich machen: ein Werk aus dem Zyklus der Deutschlandbilder des Malers und Bildhauers Anselm Kiefer aus den 1980er Jahren. Mit "Die Treppe" (Abb. 2) inszenierte der Künstler mit grob aufgetragenen Malmitteln und in einem deutlich überlebensgroßen Format eine Szene, in der eine Kolossal-Architektur mit Treppe in einem von Blitzen durchzuckten Nachthimmel aufscheint. Im Nahbereich dominieren die groben Malmittel, die kein Motiv erkennbar werden lassen. Erst in einem Abstand von etwa sechs Metern stellt es sich ein. Die steil aufsteigenden, senkrechten und diagonalen Linienzüge der Kolonnade lenken den Blick in schneller rhythmischer Folge statt in die Bildtiefe in den Bildvordergrund. Dort angekommen, lassen die wenigen farbigen Flecken im matten nachtschwarzen Himmel die Pilasterordnung riesig und das Podium mit Treppe 
davor klein und in flackernder Lebendigkeit aufstrahlen. Materialität, Größe und die Ordnung der Farben und Formen - das heißt die Komposition des Motivs bestimmen den Bildeindruck bzw. das ästhetische Erlebnis. Die Impulse bzw. das Erregungspotential, das mit den bildnerischen Mitteln stimuliert wird, befördert die Auslegung, das Motiv als eine imposante, ereignishaft aufgeladene, archaisch anmutende, ehemalige Herrschaftsarchitektur zu deuten.

Die Information, dass die Arbeit Kiefers auf der Übermalung einer vielfach vergrößerten Fotografie des für die Nationalsozialisten für Inszenierungen der Macht erbauten Zeppelinfeldes in Nürnberg beruht, versetzt der Bilderfahrung jedoch einen Stich. Denn bereits der von Walter Heges 1935 gewählte Fotoausschnitt legte den Schwerpunkt auf die Inszenierung der Macht, mit der Übernahme steigert Kiefer den Effekt ins Monumentale und setzt ihn dem Zerfall aus. Erst mit dieser Information, so zeigen es die vielfältigen Reaktionen auf das Frühwerk des Künstlers vor allem in Deutschland (im Gegensatz zum Ausland), gerät der unbedarfte, auf ästhetischen Genuss ausgerichtete Betrachter in einen Konflikt. Das mit dem Motiv in einen Zusammenhang gebrachte Erregungspotential (ästhetisches Erleben) kollidiert mit dem Wissen um den Kontext. Es forderte - und fordert noch - zu einer Antwort heraus, die bemerkenswerter Weise zunächst erneut eine affektiv-emotional geprägte ist: Faszination und Schrecken bzw. Irritation, worauf bereits Bazon Brock aufmerksam machte, bestimmten die Rezeption des Frühwerks entsprechend von Beginn an. ${ }^{78}$ In der Antwort, die Kiefer selbst gesucht hat, spiegelt sich diese Ambivalenz wider: Wie lässt sich der Wahnsinn verstehen ? $^{79}$ An diesem Beispiel zeigt sich: Es ist die Erfahrung mit dem Bild (über das Ausdrucks- bzw. ästhetische Erlebnis), die den Betrachter mit der eigenen oder - je nach kultureller Sozialisation - fremden Geschichte konfrontiert und so eine Antwort einfordert (Bildsemiotik).

Die Bilder selbst, so sollte das Beispiel abschließend verdeutlichen, können als wesentlicher Faktor der gesellschaftlichen Wirklichkeit angesehen werden. Indem sie als Meinungen, Haltungen bzw. Äußerungen über etwas verstanden werden, haben sie Anteil an der Wissensproduktion. Sie als rein ästhetische Objekte (ästhetische Theorie) oder als Dokumentationen oder Illustrationen von Geschichte zu verstehen, verbietet sich daher. Ebenso wie Sprache dienen Bilder als ein Mittel der Kommunikation von Ansichten / Meinungen eines Künstlers / Gestalters oder dessen Auftraggebers. Sie sind von deren individuellen, kulturellen und historischen Hintergründen und den Zielen, die sie verfolgen, geprägt. Mit der Bewertung bzw. Wertsetzung, die ein Motiv oder eine

78 Martina Sauer, Faszination - Schrecken. Zur Handlungsrelevanz ästhetischer Erfahrung anhand Anselm Kiefers Deutschlandbilder, in: ART-Dok. Publikationsplattform Kunstgeschichte, 05.03.2012; http://archiv.ub.uni-heidelberg.de/artdok/volltexte/2012/1851, S. $106-119$ [5.9.2014] .

79 Ebd., S. 124. 
Idee mit der Umsetzung in ein Bild erfährt und so auch verstanden werden kann, wird der Betrachter mehr oder weniger bewusst herausgefordert, dazu selbst eine Haltung einzunehmen, sei sie affirmativ oder kritisch. Das Geschichtsbild (die Ansicht des Gestalters / Künstlers), wie es sich uns über ein Bild zeigt, steht deshalb nicht fest, sondern wird ständig fortgeschrieben, da es von der Auswertung und den Reaktionen seiner Interpreten abhängt. Geschichte, wie sie ein (Meinungs-)Bild vermittelt, lässt sich derart als ein Handeln bzw. Tun beschreiben. Sie ist durch ein Setzen (des Gestalters / Künstlers), ein Erleben (des Betrachters) und Weiterleben (in der Reaktion des Betrachters) gekennzeichnet. Mit der Antwort, die ein Bild einfordern kann, erweist sich das Bild selbst als ein Akteur in der Geschichte bzw. im Leben. Es lebt im Betrachter und seinen Reaktionen und Handlungen weiter. Dabei können die möglichen Antworten auch systemimmanent erfolgen, indem das Bild reproduziert und eventuell variiert wird. Kiefer selbst hat durch die Verwendung von Heges' Fotovorlage diesen Weg beschritten. Mit der Formulierung von Ansichten über ein Bild werden jedoch nicht nur Wertesysteme (Meinungsbilder) konstituiert, die als solche angenommen oder verworfen werden können, sondern sie werden über die jeweiligen Antworten bzw. Praktiken zugleich tradiert und je nachdem verändert. So kann ein Bild als ein historisch variabler Handlungsraum angesehen werden, der sich in ständigem Wandel befindet. Bilder und das Verständnis, das sie uns jeweils von der Lebenswelt vermitteln, lassen sich vor diesem Forschungshintergrund als ein System der Verständigung und des Austauschs von Werten begreifen. Die Kommentierung eines Zusammenhangs durch ein Bild, die Weitergabe von Informationen oder deren Veränderung sorgen dafür, dass Bilder als konkrete Faktoren unserer gesellschaftlichen Wirklichkeit ständig unter uns zirkulieren. Die Möglichkeiten der neuen Techniken forcieren diesen Impuls noch. Doch erst über den Einbezug der Leiblichkeit bzw. des affektivemotional realen Faktors, den Bildwerke transportieren und den wir verstehen, lässt sich ein solches weitgefasstes und tiefgreifendes Konzept vom Bild als Mittel der Kommunikation und zugleich als historischem Akteur vorstellen. Bilder als solche Akteure ernst zu nehmen, fordert die historische Bildkunde und -forschung heraus, ihre Methoden und Ergebnisse zu überdenken. Die Neubewertung der klassischen Formanalyse spielt dabei eine entscheidende Rolle. Statt Wesensschau (ästhetische Theorie) oder Mentalitätsgeschichte (Stilgeschichte) - was ihr gerne unterstellt wird - erlaubt sie vor dem Hintergrund der ausgewerteten bildanthropologischen Forschungen, das Potential von Bildern zur kulturellen Wertebildung und damit ihre elementare Handlungsrelevanz herauszuarbeiten. 